respectively, $\mathrm{p}=0.016$; ISRs: $17.31 \%, 95 \% \mathrm{Cl} 14.25-20.83 \%$ and $22.36 \%$, $95 \% \mathrm{Cl} 20.82-23.99$, respectively, $\mathrm{p}=0.004)$. The difference between PBO- and ETN-treated patients was statistically significant in the 2 age groups combined (Breslow Day interaction) for angioedema ( $p=0.003)$, ISRs $(p=0.042)$, and NMSC $(p=0.042)$. Older, but not younger, patients had greater (but not statistically significant) occurrence of $\mathrm{CHF}, \mathrm{SI}$, and NMSC when taking ETN vs PBO. There was an interesting, non-significant, reduction of ILD occurrence in older, but not younger ETN-treated vs PBO-treated patients (figure 1). There were no significant agerelated increases in the occurrence of other AEs of interest such as uveitis, opportunistic infections (invasive fungal, protozoal, bacterial, and atypical mycobacterial infections and Legionella), tuberculosis, lymphoma, melanoma, or leukaemia. The smaller number of patients in the older cohort was a study limitation, and further stratification of the data in the higher age brackets may provide key information on the age at which the risk of particular AEs increases.

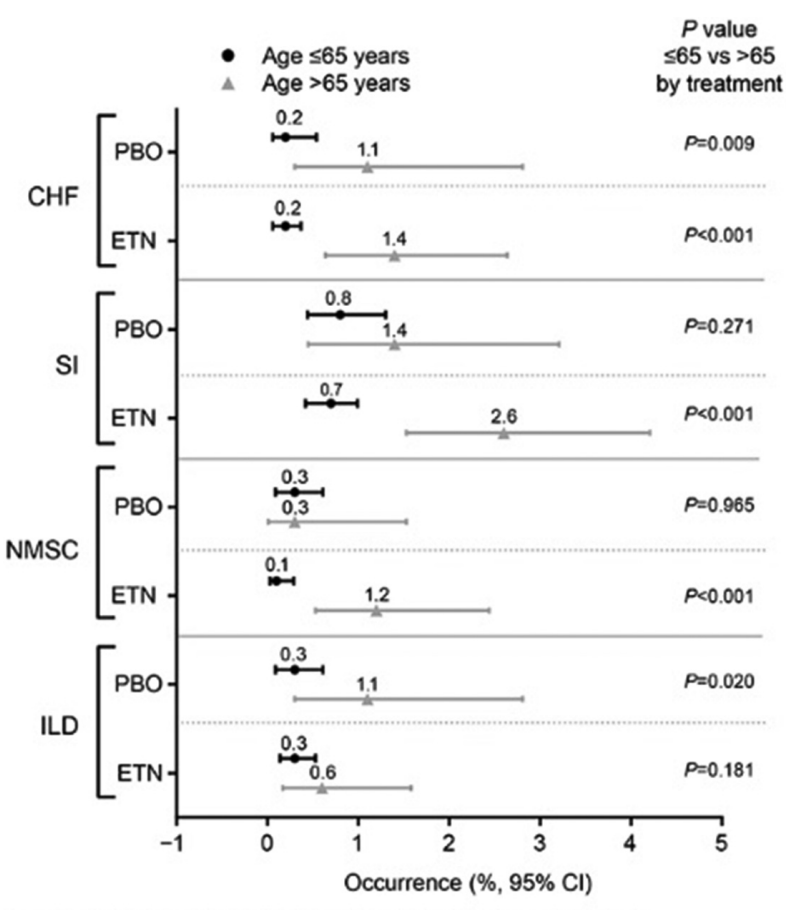

SI included pneumonia, cellulitis, septic arthritis, sepsis, and parasitic infection.

Abstract FRI0055 - Figure 1. Occurrence of AEs with notable age-related differences in patients with RA

SI included pneumonia, cellulitis, septic arthritis, sepsis, and parasitic infection. Conclusions: The occurrence of $\mathrm{CHF}, \mathrm{SI}$, and NMSC was higher in older vs younger patients taking ETN. While the analysis suggests an overall acceptable safety profile of ETN, the relative risks and benefits need to be considered when treating older patients.

Disclosure of Interest: C. J. Edwards Grant/research support from: Pfizer, Abbvie, Biogen, UCB, Janssen, Samsung, Mundipharma, Roche, Lilly, Merck, Consultant for: Pfizer, Abbvie, Biogen, UCB, Janssen, Samsung, Mundipharma, Roche, Lilly, Merck, Speakers bureau: Pfizer, Abbvie, Biogen, UCB, Janssen, Samsung, Mundipharma, Roche, Lilly, Merck, K. Roshak: None declared, J. Bukowski Shareholder of: Pfizer, Employee of: Pfizer, R. Pedersen Shareholder of: Pfizer, Employee of: Pfizer, M. Thakur Shareholder of: Pfizer, Employee of: Pfizer, L. Marshall Shareholder of: Pfizer, Employee of: Pfizer, H. Jones Shareholder of: Pfizer, Employee of: Pfizer

DOI: 10.1136/annrheumdis-2018-eular.2414

\section{FRI0056 \\ CLINICAL FEATURES AT THE ONSET OF LYMPHOPROLIFERATIVE DISORDER IN PATIENTS WITH RHEUMATOID ARTHRITIS}

D. Kobayashi ${ }^{1,2}$, S. Ito ${ }^{2}$, Y. Wada ${ }^{1}$, I. Narita ${ }^{1}$, K. Nakazono ${ }^{2} .{ }^{1}$ Division of Clinical Nephrology and Rheumatology, Niigata University Graduate School of Medical and Dental Sciences, Niigata; ${ }^{2}$ Rheumatology, Niigata Rheumatic Center, Shibata, Japan

Background: Rheumatoid arthritis (RA) is intrinsically associated with an increased incidence of lymphoproliferative disorders (LPDs). Furthermore, treatment with immunosuppressing agents, such as methotrexate (MTX) sometimes leads to the development of immunosuppressing agents related LPDs. Thus the development of LPDs is an increasingly important issue. However, previous studies focused on the pathological features and regression of LPDs after ceasing MTX administration, and evidence that could allow for the early diagnosis of LPDs in patients with RA is lacking.

Objectives: We surveyed the clinical course of patients with RA who developed LPDs at our hospital, with a focus on the clinical course and laboratory findings leading to their development.

Methods: Patients with RA who were treated at Niigata Rheumatic Centre between April

2011 and December 2017 were analysed. Patient data were obtained retrospectively from medical records. Twenty-nine patients (14 men, 15 women) developed a LPD (LPD group). We compared them with 87 patients without LPD (non-LPD group), who were matched for age, sex, and RA duration among 2628 patients with RA who attended our outpatient department in 2017. Data indicated are median $\left(\mathrm{IQR}_{1 / 4}-3 / 4\right)$ values.

Results: The median patient age was 71.0 (64.0-74.0) years, and the duration of RA was 16.0 (10.0-22.0) years in both groups. Twelve patients were diagnosed with diffuse large $B$ cell lymphoma, 5 with Hodgkin lymphoma, and 4 with $T$ cell lymphoma; 8 patients had other diagnosis.

Of 29 patients, 23 were treated with MTX, 10 with tacrolimus, 8 with biological disease-modifying antirheumatic drugs (bDMARD). Twenty-two patients had Steinbrocker stage-IV RA. Multivariate analysis showed that Steinbrocker stage IV and MTX treatment were risk factors for the development of LPDs, with hazard ratios of 4.65 and 3.32 , respectively.

In the LPDs group, although the C-reactive protein level (CRP) level and lactate dehydrogenase (LDH) level significantly rose (CRP, 0.10 [0.090-0.20] mg/dL 6 months before LPD diagnosis to 1.9 [1.1-3.8] $\mathrm{mg} / \mathrm{dL}$ at LPD diagnosis, LDH, 198.0 [184.0-230] to 310 [210-489] IU/L), neither the tender joint count (TJC) and swollen joint count (SJC), nor matrix metalloproteinase-3 (MMP-3) level showed any significant change. On the contrary, CRP and LDH levels, as well as TJC, SJC, and MMP-3 level did not change significantly at the 6 month follow-up in non-LPDs group. LDH, MMP, and CRP ratios were calculated as the ratio of them at LPD diagnosis to those at 6 months before diagnosis. CRP and LDH ratios were significantly higher in the LPD group (CRP, 1.00 [0.65-1.75] in the non-LPD group vs 9.50 [2.40-23.0] in the LPD group; LDH, 1.00 [0.93-1.09] in the non-LPD group vs 1.46 [1.17-2.04] in the LPD group). However, the MMP-3 ratio was not different significantly between the non-LPD and LPD groups (1.00 [0.88-1.12] vs 0.98 [0.85-1.21]). MMP-3 ratio/CRP ratio was significantly lower in the LPD group than in the non-LPD group $(0.0979$ [0.036-0.384] vs 1.00 [0.54-1.67]). The receiver operating characteristic curve suggested that MMP-3 ratio/CRP ratio $<0.476$ was the best cut-off point for the prediction of LPD development, with a sensitivity of $90.9 \%$, specificity of $79.0 \%$, and an area under the curve of 0.894 .

Conclusions: LDH elevation, and CRP elevation that is disproportionate to TJC, SJC, and MMP-3 level might indicate underlying LPD.

Disclosure of Interest: None declared

DOI: 10.1136/annrheumdis-2018-eular.2454

\section{FRI0057 EFFECTS OF ANTI-TNF THERAPY ON VASCULAR BIOMARKER LEVELS IN RHEUMATOID ARTHRITIS}

E. Végh ${ }^{1}$, A. Hamar ${ }^{1}$, Á. Horváth ${ }^{1}$, S. Szántó ${ }^{1}$, G. Szücs ${ }^{1}$, A. Pusztai ${ }^{1}$, A. Domján ${ }^{1}$, K. Hodosi ${ }^{1}$, G. Kerekes ${ }^{2}$, R. Gesztelyi ${ }^{3}$, J. Zsuga ${ }^{4}$, Z. Prohászka ${ }^{5}$, Z. Szekanecz ${ }^{1}$. ${ }^{1}$ Department of Rheumatology; ${ }^{2}$ Intensive Care Unit, Department of Internal Medicine; ${ }^{3}$ Department of Pharmacology and Pharmacotherapy, University of Debrecen, Faculty of Medicine; ${ }^{4}$ Department of Healthy Systems Management, University of Debrecen, Faculty of Public Health, Debrecen; ${ }^{5}$ Third Department of Internal Medicine, Semmelweis University, Faculty of Medicine, Budapest, Hungary

Background: Prevoius studies have shown an increased risk of cardiovascular disease in rheumatoid arthritis (RA), due to RA-associated inflammation. Different vascular biomarkers, such as anti-hsp65 antibodies, asymmetric dimethylarginine (ADMA) and B-type natriuretic peptide (BNP) have been associated with atherosclerosis and RA. Anti-hsp65 antibodies are also linked also to autoimmunity, inflammation and atherosclerosis. ADMA is an endogenous competitive inhibitor of NOS and consequential can lead to increased nitrosative and oxidative stress. ADMA has been implicated with atherosclerosis, and in RA. BNP is also involved in cardiovascular diseases.

Objectives: The aim of this study was to assess the effects of anti-TNF therapy on different vascular biomarkers, such as anti-hsp65, ADMA and BNP in patients with RA and their correlation with different laboratory and clinical markers.

Methods: Altogether 36 RA patients were recruited and treated with either etanercept (ETN) or certolizumab pegol (CZP) in this 12 months follow-up study Assessments were performed at baseline, at month 6 and 12. Amounts of IgG antibodies reacting with recombinant M. bovis hsp65 (Lionex, Braunschweig, Germany) were measured by ELISA. Asymmetric dimethylarginine (ADMA) was assesed by HPLC with fluorescent detection. BNP fragments were assessed by commercially avaiable ELISA kit (Biomedica, Vienna). In addition, disease activity 